\title{
Molecular analysis of cytosolic and mitochondrial malate dehydrogenases isolated from domestic cats (Felis catus)
}

\author{
N. Sasaki ${ }^{1}$, M. Nakamura ${ }^{1}$ and S. Soeta ${ }^{2}$ \\ ${ }^{1}$ Laboratory of Veterinary Biochemistry, School of Veterinary Medicine, \\ Nippon Veterinary and Life Science University, Tokyo, Japan \\ ${ }^{2}$ Laboratory of Veterinary Anatomy, School of Veterinary Medicine, \\ Nippon Veterinary and Life Science University, Tokyo, Japan \\ Corresponding author: N. Sasaki \\ E-mail: noris@nvlu.ac.jp
}

Genet. Mol. Res. 13 (3): 6855-6864 (2014)

Received July 8, 2013

Accepted January 4, 2014

Published August 29, 2014

DOI http://dx.doi.org/10.4238/2014.August.29.7

\begin{abstract}
Malate dehydrogenase (MDH) plays crucial roles in energy and cellular metabolism. In this study, we describe the identification and characterization of cytosolic MDH (MDH1) and mitochondrial MDH (MDH2) in liver of domestic cat (Felis catus). To clone the feline full-length $M D H$ genes, we performed rapid amplification of cDNA ends. The $M D H 1$ gene encoded a protein of 334 amino acids and the $M D H 2$ gene encoded a protein of 338 amino acids, containing a 24-amino acid mitochondrial target sequence. The feline $\mathrm{MDH} 1$ and $\mathrm{MDH} 2$ proteins shared, respectively, 98.8-93.7 and 96.7-94.4\% homology with dog, giant panda, horse, cow, pig, human, mouse, and rat. The feline MDHs had a highly conserved active motif, which contained important residues for catalysis and coenzyme binding. The putatively acetylated lysine residues that regulate MDH activity were also conserved at K118, K121, and K298 in MDH1, and $\mathrm{K} 185, \mathrm{~K} 301, \mathrm{~K} 307$, and K314 in MDH2. Both MDH1 and MDH2
\end{abstract}


mRNAs were ubiquitously expressed, but these expression levels varied in a tissue-specific manner. Both $M D H$ genes were expressed at considerably high levels in heart and skeletal muscle, but at low levels in lung and spleen.

Key words: Cytosolic malate dehydrogenase; Lysine acetylation; Mitochondrial malate dehydrogenase; Domestic cat; Felis catus

\section{INTRODUCTION}

Nicotinamide adenine dinucleotide (NAD)-dependent malate dehydrogenase (MDH; EC1.1.1.37) catalyzes the reversible conversion of malate to oxaloacetate linked to oxidation and reduction of the coenzyme NAD. The detailed aspects of MDH have been discussed in several reviews (Goward and Nicholls, 1994; Musrati et al., 1998; Minárik et al., 2002). Most eukaryotes express two types of MDH, cytosolic MDH (MDH1) and mitochondrial MDH (MDH2). The MDH enzymes are essential for both gluconeogenesis and the tricarboxylic acid (TCA) cycle and therefore play crucial roles in energy metabolism. MDH1 is the key enzyme in the malate/aspartate shuttle, which is the dominant pathway in the liver and heart (Scholz et al., 1998), while MDH2 is known to be a key enzyme in the TCA cycle. Cytosolic MDH, mentioned as MDH2 in yeast, has been shown to work as a gluconeogenic enzyme, because it is required for the growth of yeasts on a minimal medium in the presence of gluconeogenic precursors (Gibson and McAlister-Henn, 2003).

Feline liver requires the high enzymatic activities of MDHs to produce glucose via gluconeogenesis. As domestic cats (Felis catus) rely mainly on hepatic glucose production to maintain blood glucose levels, gluconeogenesis from amino acids is highly constitutive even after eating (MacDonald et al., 1984).

Cellular metabolic rate and glucose utilization status affect the expression and activity of MDHs. For example, MDH1 expression level in leukocytes is lower in cats than in dogs (Washizu et al., 2002). In addition, MDH activity and expression is decreased in diabetes (Magori et al., 2005), suggesting that this glucose metabolic disorder affects its expression and/ or activity in cats. However, the structure, regulatory mechanisms, and distribution of feline MDHs are still unclear.

Recent proteomic studies have shown that lysine acetylation regulates many metabolic enzymes involved in glycolysis, gluconeogenesis and fatty acid synthesis, as well as the TCA and urea cycles (Kim et al., 2006; Choudhary et al., 2009; Wang et al., 2010; Zhao et al., 2010). MDH activity is regulated by protein acetylation, which is a crucial post-transcriptional regulator of energy and cell metabolism. Zhao et al. (2010) demonstrated that MDH2 activation is not observed when putative lysine residues are replaced by arginine. We speculated, therefore, that the activity of feline $\mathrm{MDH}$ is regulated similarly by lysine acetylation and deacetylation.

The aims of this study were: 1) to identify the primary structure of feline MDHs; 2) to explore the functional active motif and putative lysine acetylation residues; and 3) to determine the expression patterns of $M D H$ mRNAs in feline tissues. Our findings provide a foundation for further functional analyses of feline MDHs. 


\section{MATERIAL AND METHODS}

\section{RNA extraction and partial-cDNA cloning}

Feline liver RNA was extracted with TRI Reagent (Sigma-Aldrich, St. Louis, MO, USA) and then purified using Oligotex-dT30 < Super $>$ (Takara Bio Inc., Otsu, Shiga, Japan). First-strand cDNA was synthesized (SuperScript II reverse transcriptase; Invitrogen, Carlsbad, CA, USA) with oligo d(T) as the primer. To obtain partial cDNAs of $M D H$, polymerase chain reaction (PCR) primers were designed considering the canine MDH1 (XP 531844) and MDH2 (XP_849944) mRNA sequences (Table 1). Reverse transcription PCR (RT-PCR) was performed with feline liver cDNA as the template using these primers. The PCR products were ligated into the pT7Blue T-vector (Novagen, Inc., Madison, WI, USA) and then transformed into NovaBlue competent Escherichia coli cells (Novagen). The inserts were sequenced using BigDye Terminator v3.1 and an ABI PRISM 310 DNA Sequencer (Applied Biosystems, Foster City, CA, USA).

\section{Full-length cDNA cloning of feline MDH1 and MDH2}

After determining partial MDH fragments, the rapid amplification of cDNA ends (RACE) method was used to clone full-length feline $M D H$ cDNA, using the GeneRacer kit (Invitrogen) according to the manufacturer protocol. One of the 3'-RACE primers for MDH1 (MDH1-for2; Table 1) was designed on the basis of a partial sequence. Reactions for 5'- and 3'-RACE were performed with GeneRacer primers and gene-specific primers (Table 1). The amplified PCR products were purified and subcloned. Both strands of the longest 5'-RACE and 3'-RACE cDNA inserts were sequenced using the primer walking method.

\begin{tabular}{|c|c|c|c|}
\hline Primer & Sequence $\left(5^{\prime}-3^{\prime}\right)$ & Gene & Aplication \\
\hline MDH1-for & AGC CAA TAC CAA CTG CCT GAC TGC & $\mathrm{MDHI}$ & RT-PCR \\
\hline MDH1-rev & GGA GAT AAT GCC CAT GGA CAC AAA & MDHI & RT-PCR, 5'-RACE \\
\hline MDH1-rev2 & AAT GTC TGC TGC AAA AGC CAT CTC & $M D H 1$ & 3'-RACE \\
\hline MDH2-for & GCA TGA CAC GGG ATG ATC TGT TC & $\mathrm{MDH} 2$ & RT-PCR, 3'-RACE \\
\hline MDH2-rev & CAC TGA GAG ATC AGG GGG ATG ATG G & $\mathrm{MDH} 2$ & RT-PCR, 5'-RACE \\
\hline GeneRacer oligo d(T) primer & GCT GTC AAC GAT ACG CTA CGT AAC GGC ATG ACA GTG (T)18 & & RT \\
\hline GeneRacer 5'-primer & CGA CTG GAG CAC GAG GAC ACT GA & & 5'-RACE \\
\hline GeneRacer 3'-primer & GCT GTC AAC GAT ACG CTA CGT AAC G & & 3'-RACE \\
\hline qMDH1-for & AAG ATG TTG CCT TCA AAG ACC T & $M D H 1$ & qPCR \\
\hline qMDH1-rev & TTC CCA CCA CAA TAA CCT TAA CTG & $M D H I$ & qPCR \\
\hline qMDH2-for & CAA GAA GCA TGG AGT ATA CAA CCC & $\mathrm{MDH} 2$ & qPCR \\
\hline qMDH2-rev & ACC TTG GGA GTA CAC TGA GAG & $\mathrm{MDH} 2$ & qPCR \\
\hline qCYPA-for & CAA AGT TCC CAA GAC AGC AGA GA & CYPA & qPCR \\
\hline qCYPA-rev & AGT GCC ATT ATG GCG TGT GAA & CYPA & qPCR \\
\hline
\end{tabular}

\section{Sequence analyses}

A search for the mitochondrial targeting sequence in $\mathrm{MDH} 2$ was conducted using the MitoProt program (http://ihg.gsf.de/ihg/mitoprot.html; Claros and Vincens, 1996). Active mo- 
tif searches were performed against the PROSITE motif database via the MOTIF search program (Falquet et al., 2002) available on the website of the Kyoto Encyclopedia of Genes and Genomes (http://www.genome.jp/tools/motif/MOTIF.html). Alignment of multiple sequences was performed using the ClustalW program.

The protein sequence reference numbers used in the alignment analysis of MDH1 and MDH2 are respectively as follows: dog (XP_531844, XP_849944); giant panda (XP_002921852, XP_002923512); horse (XP_001494315, NP_001182455); cow (NP_001029800, AA109598); pig (NP_999039, NP_00121082); human (NP_005908, NP_005909); rat (NP_150238, NP_112413); and mouse (NP_032644, NP_032643).

\section{Determination of tissue distribution by quantitative real-time PCR (qPCR)}

Tissue distribution of MDH1 and MDH2 mRNA was determined by qPCR. Total RNAs from various feline tissues, including heart, lung, liver, pancreas, stomach, ileum, colon, kidney, spleen, skeletal muscle, and adipose tissue were purchased from Zyagen (San Diego, CA, USA). The relative expression of $M D H 1, M D H 2$, and cyclophilin A (CYPA) mRNAs were determined by qPCR. The primers for qPCR are shown in Table 1 . The primers used for CYPA were reported in the study by Zini et al. (2010). First-strand cDNA was synthesized (PrimeScript RT Reagent kit, Takara Bio) and the subsequent qPCR was carried out on a GeneAmp 7500 Sequence Detection System (Applied Biosystems) with SYBR Premix Ex Taq II (Takara Bio) following the manufacturer protocol. The comparative $\mathrm{Ct}$ method ( $\Delta \Delta^{\mathrm{Ct}}$ method) was used to analyze the relative fold-changes of MDHs (Bookout and Mangelsdorf, 2003). Expression levels of $M D H 1$ and $M D H 2$ in each tissue were normalized to CYPA. The ratios of $M D H 1 / C Y P A$ and $M D H 2 / C Y P A$ from each tissue were standardized to the liver ratio of 1.0-fold. Each sample was analyzed in duplicate and the experiment was repeated three times.

\section{RESULTS}

\section{Primary structures of feline MDH1 and MDH2}

The nucleotide and deduced amino acid sequences of the feline $M D H 1$ cDNAs are shown in Figure 1A. Feline MDH1 consists of $1292 \mathrm{bp}$, which includes a 5'-untranslated region (UTR) of $75 \mathrm{bp}$ and a 3'-UTR of $212 \mathrm{bp}$ (Figure 1A). Feline MDH1 cDNA contains a polyadenylation signal (AATAAA) $21 \mathrm{bp}$ upstream from the poly(A) tail. This cDNA encodes a polypeptide of 334 amino acids (aa). The calculated molecular mass of the polypeptide is $36.4 \mathrm{kDa}$. The complete feline $M D H 1$ nucleotide sequence was submitted to the DNA Data Bank of Japan (DDBJ) as accession number AB113364.

The nucleotide and deduced amino acid sequences of feline $M D H 2$ cDNA are shown in Figure 1B. Feline MDH2 cDNA consists of $1281 \mathrm{bp}$, including a 5'-UTR of $81 \mathrm{bp}$ and a 3'-UTR of $180 \mathrm{bp}$ (Figure 1B). Feline $M D H 2$ cDNA contains a polyadenylation signal $26 \mathrm{bp}$ upstream from the poly(A) tail. Feline $M D H 2$ encodes a polypeptide of 338 aa with a calculated molecular mass of $35.5 \mathrm{kDa}$. The feline $\mathrm{MDH} 2$ nucleotide sequence was deposited in the DDBJ as accession number AB751611. 
A

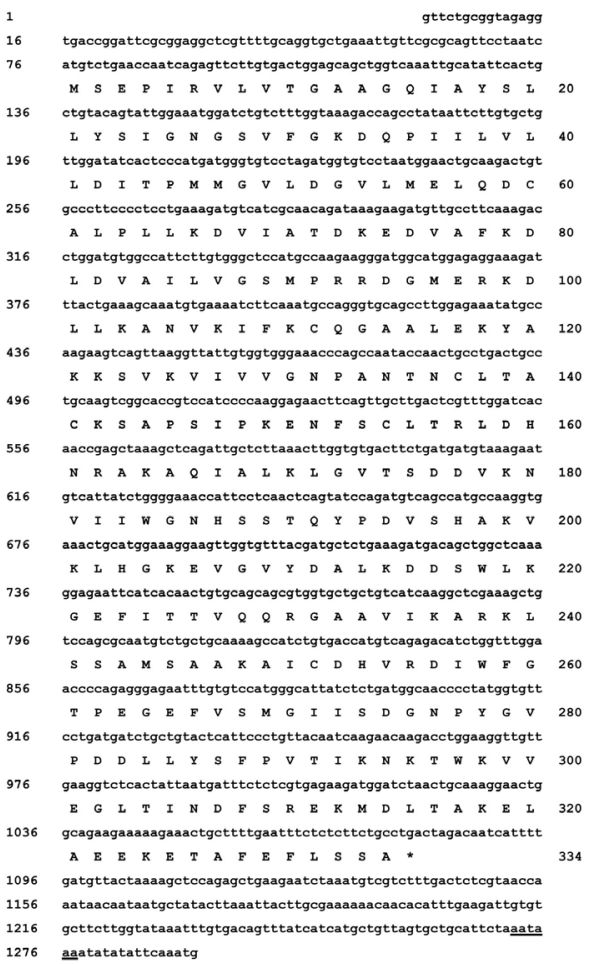

B

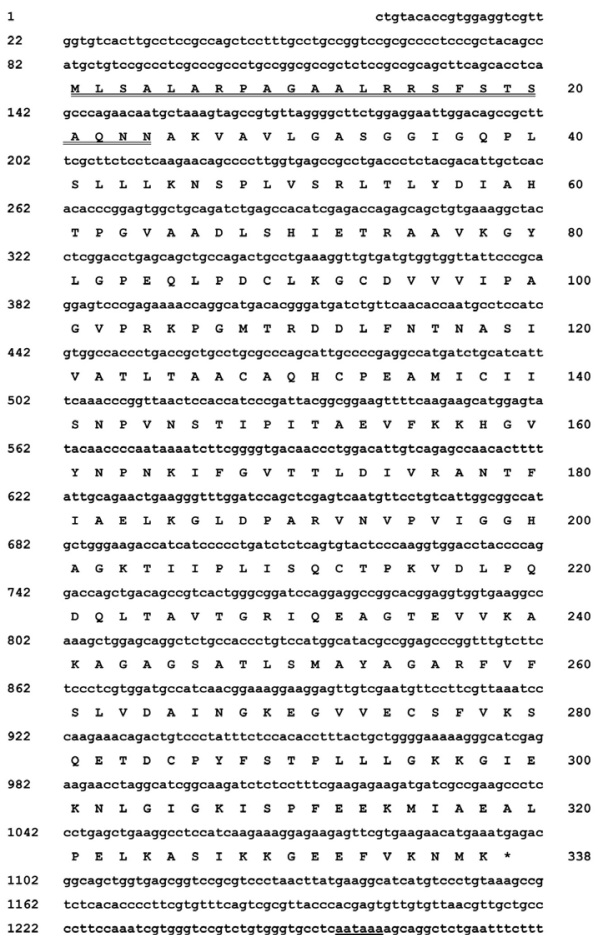

Figure 1. Nucleotide and deduced amino acid sequences of feline MDH1 and MDH2. The nucleotide sequence of the $M D H$ cDNA is shown on the top line, and its predicted amino acid sequence is shown below in one-letter code. Numbers on the left and right refer to the nucleotide and amino acid residue, respectively. The asterisk indicates the translation termination codon. The poly(A) signal (AATAAA) is underlined. The double underline indicates a putative mitochondrial-targeting signal. A. MDH1; B. MDH2.

\section{Sequence analyses of active motifs and essential residues for MDH}

Organelle-specific MDHs, such as mitochondrial, peroxisomal, chloroplast, and glyoxysomal MDHs, are translated as precursor proteins that possess a specific organellar targeting signal sequence at their amino terminal ends (Gietl, 1992). MitoProt analysis showed that the putative feline $\mathrm{MDH} 2$ precursor possesses a mitochondrial targeting signal of 24-aa residues at its amino terminal end, indicating that the mature MDH2 comprises 314-aa residues with a calculated molecular mass of $33.0 \mathrm{kDa}$.

Computational analysis by the motif-search algorithm confirmed that feline MDH1 and MDH2 have crucial, highly conserved amino acid residues that participate in catalysis, coenzyme binding, and substrate binding. MDH possesses a conserved active motif called the MDH active site signature (PROSITE, PS00068): [LIVM]-T-[TRKMN]-L-D-x(2)-R-[STA]$\mathrm{x}(3)-[L I V M F Y]$. This motif was found in feline MDH1 (residues 155-167) as L-T-R-L-D-hnR-A-kaq-I and in feline MDH2 (residues 169-181) as V-T-T-L-D-iv-R-A-ntf-I (Figure 2). The motif-search also revealed that several important residues for catalysis and coenzyme binding 
are conserved. The aspartate residues at D42 (MDH1) and D57 (MDH2) are essential for coenzyme binding and specificity for the adenosine ribose hydroxyl group (Birktoft et al., 1989; Hall et al., 1992; Kelly et al., 1993). Three arginine residues (R92, R98, and R162 in MDH1, and R104, R110, and R176 in MDH2) are highly conserved among many MDHs and are important for substrate binding and catalysis. MDH enzymes require a histidine/aspartate pair to form a charge relay system (Goward and Nicholls, 1994). The feline MDHs have the corresponding histidine/aspartate pair at H187/D159 (MDH1) and H200/D173 (MDH2).

A

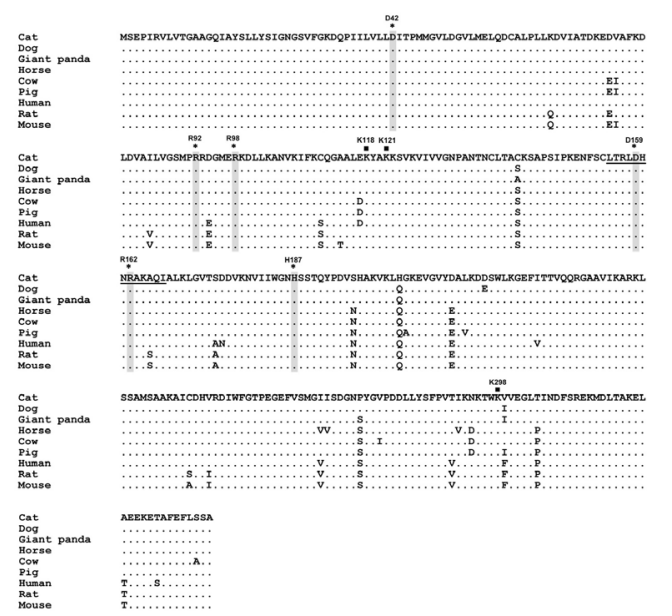

B

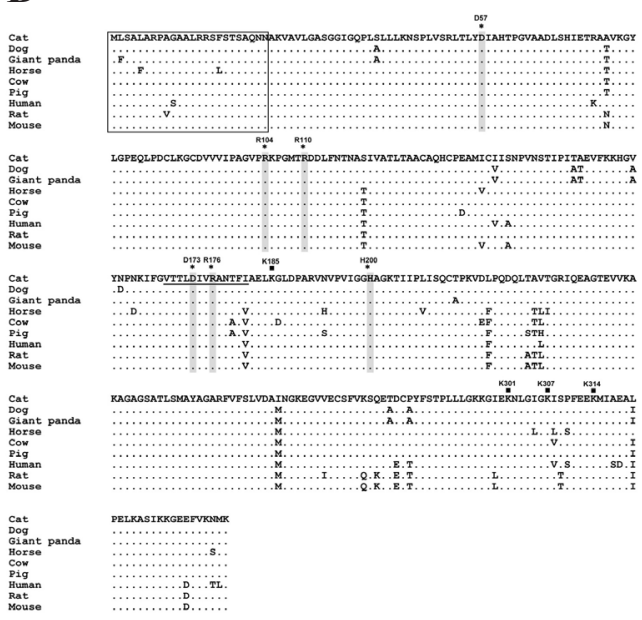

Figure 2. Multiple alignment of the amino acid sequences of feline MDH1 and MDH2. Identical residues across all sequences are represented by dots. The line under the MDH sequence indicates the MDH active motif, while asterisks above the sequence indicate residues that are important for catalysis and coenzyme binding. Solid square indicates a putative lysine acetylation site. Mitochondrial target sequences are boxed. The alignment was produced using ClustalW. A. MDH1; B. MDH2.

MDH activity by lysine-acetylation has been well studied, and several recent reports have shown that many metabolic enzymes are regulated by lysine acetylation (Kim et al., 2006; Choudhary et al., 2009; Wang et al., 2010; Zhao et al., 2010). Putative lysine acetylation residues are conserved in both MDH1 and MDH2: at K118, K121, and K298 in feline MDH1 (Figure 2A) and K185, K301, K307, and K314 in MDH2 (Figure 2B).

\section{Comparison of MDH amino acid sequences from cats and other mammals}

To search for species-specific differences, we compared the amino acid sequences of feline MDH1 and MDH2 with those of selected mammalian species. As shown in Table 2, feline MDH1 shares 98.8-93.7\% sequence homology with MDH1 from other mammals. Protein sequence alignment revealed that feline MDH1 shares the highest homology (98.8\%) with canine MDH1 and giant panda MDH1, while it shares the lowest homology with murine MDH1 (93.7\%). Feline MDH2 shares the highest and lowest homology with canine (96.7\%) and human (94.4\%) MDH2, respectively (Table 2). In contrast, the sequence homology between feline MDH1 and MDH2 was found to be only $16.8 \%$. 
Table 2. Amino acid sequence comparisons between feline MDHs and those of other mammals.

\begin{tabular}{lccccccccc}
\hline & Cat & Dog & Giant panda & Horse & Cow & Pig & Human & Rat & Mouse \\
\hline MDH1 & 100 & 98.8 & 98.8 & 97.0 & 96.4 & 96.1 & 94.9 & 94.3 & 93.7 \\
MDH2 & 100 & 96.7 & 96.4 & 94.7 & 96.4 & 96.4 & 94.4 & 94.7 & 94.7 \\
MDH1 vs MDH2 & 16.8 & 15.6 & 15.6 & 16.5 & 15.6 & 15.9 & 15.3 & 15.6 & 15.3 \\
\hline
\end{tabular}

Data are reported as percent.

\section{Determination of tissue distribution by qPCR}

We performed qPCR to determine the expression of $M D H 1$ and $M D H 2$ mRNA in different feline tissues. The constitutively expressed CYPA gene was used as an internal control. The results indicated that $M D H 1$ and $M D H 2$ mRNAs are expressed ubiquitously in all tissues tested (Figure 3). In normal cats, MDH1 mRNA expression levels, normalized to liver (1.0fold), are the highest in skeletal muscle (39.9-fold) and heart (25.4-fold) (Figure 3A); moderate in kidney (6.3-fold), stomach (4.2-fold), pancreas (2.6-fold), and ileum (1.4-fold); and the lowest in spleen ( 0.32 -fold), colon ( 0.70 -fold), lung ( 0.72 -fold), and adipose tissue $(0.78$-fold) (Figure 3B). $M D H 2$ mRNA expression levels are the highest in skeletal muscle (76.7-fold) and heart (9.9-fold) (Figure 3A), moderate in ileum (2.4-fold), kidney (1.8-fold), adipose tissue (1.4-fold), and colon (1.1-fold), and the lowest in lung (0.26-fold), spleen (0.51-fold), pancreas (0.45-fold), and stomach (0.72-fold) (Figure 3B).
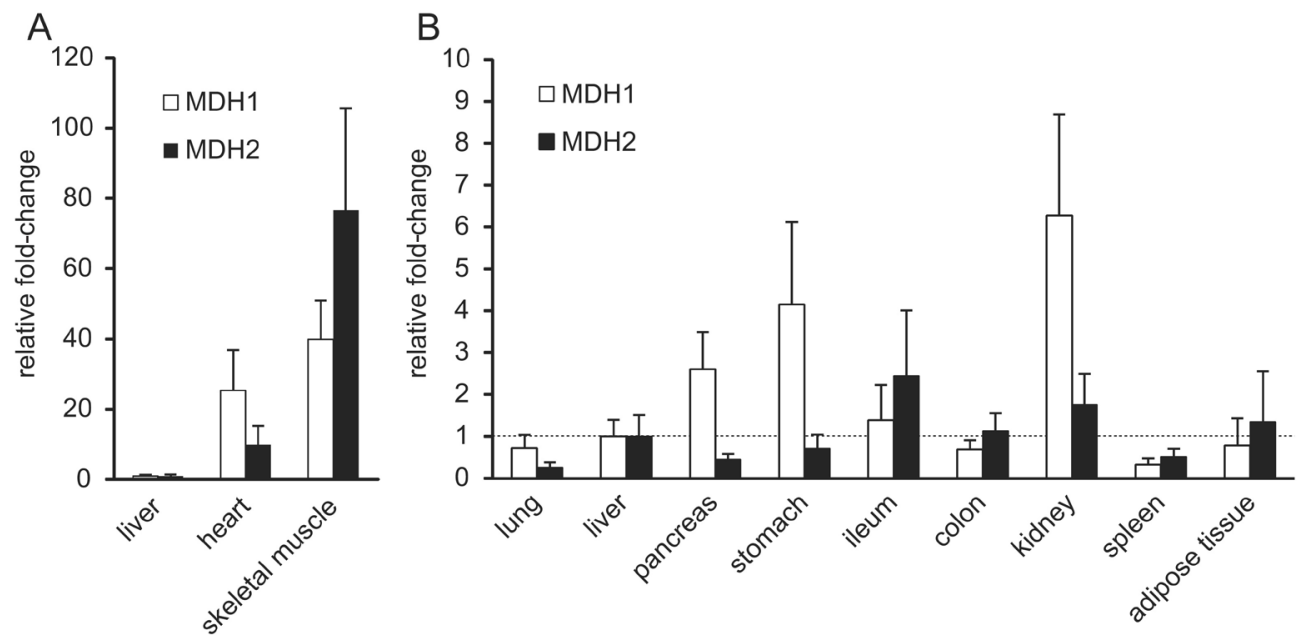

Figure 3. Expression of malate dehydrogenase mRNAs in tissues by quantitative RT-PCR. MDH1 mRNA (open columns) and $M D H 2$ mRNA (closed columns) expression levels in each tissue are plotted as averages of the foldchanges. The expression level of cyclophilin A (CYPA) mRNA was used as an internal control. The ratio of $M D H$ mRNA to CYPA mRNA (expressed as arbitrary units) from each tissue was normalized to liver. The columns represent the mean and standard deviation calculated from results of three independent qPCRs. A. High MDH expression levels in heart and skeletal muscle. B. Moderate or low MDH expression levels in tissues.

\section{DISCUSSION}

MDH is one of the most well-studied enzymes because of its crucial roles in the cell me- 
tabolism. However, the structure, regulatory mechanisms, and distribution of feline MDHs have not been reported. In this study, we cloned two full-length $M D H$ cDNAs from cats by RACE and determined their primary structure.

The cDNAs obtained were identified as feline $M D H 1$ and $M D H 2$ cDNA according to their homology to $M D H$ genes of other species. The deduced polypeptide sequences of feline MDH1 and MDH2 show high homology with their counterpart in other mammals, including dog, giant panda, horse, cow, pig, human, mouse, and rat.

All active motifs and amino acid residues, which are conserved in both MDHs from other species, are important for catalysis, coenzyme binding, and substrate binding (Musrati et al., 1998). As shown in Table 2, the results indicate that the primary structures of MDHs are highly conserved among mammals; furthermore, they lack specific substitutions or deletions. The sequence homology between feline MDH1 and MDH2 is only $16.8 \%$; this finding is consistent with that of other mammals (Table 2) from previous reports (Joh et al., 1987; McAlister-Henn, 1988). Our results show that feline MDH1 is more closely related to MDH1 from other mammals than feline MDH2. We presume that feline MDH1 and $\mathrm{MDH} 2$ have similar activities and are expressed in the cytoplasm and mitochondria, respectively.

More recently, Zhao et al. (2010) reported that lysine acetylation plays a major role in metabolic regulation in humans. They reported that $\mathrm{MDH}$ is activated by acetylation of lysine residues, and found the presence of four acetylated lysine residues (K185, K301, $\mathrm{K} 307$, and K314) in human MDH2. Our findings showed that the corresponding lysine residues are conserved in feline $\mathrm{MDH} 2$ at the same positions (Figure 2B). Kim et al. (2012) reported that the acetylation level of MDH1 increases during adipogenesis in adipocytes. When putative lysine residues are replaced with arginine, decreased activity of the mutated enzyme results in the inhibition of adipogenesis. On the basis of the feline MDH1 sequence, we determined that putative lysine acetylation residues are conserved and located at K118, K121, and K298 in feline MDH1 (Figure 2A). These findings suggest that the activity of feline MDH is similarly regulated by lysine acetylation and deacetylation.

In humans, MDH1 mRNA is expressed at relatively high levels in heart, skeletal muscle and brain (Lo et al., 2005). Similarly, Mori et al. (2009) reported that MDH1 mRNA is highly expressed in skeletal muscle in cats. We confirmed the high expression level of MDH1 in feline heart and skeletal muscle and determined that MDH2 is also expressed at high levels in heart and skeletal muscle. High expression levels of MDH1 and MDH2 in heart and skeletal muscle are reasonable because these tissues have a high metabolic rate and mainly use the malate/aspartate shuttle, which uses MDH1 and MDH2 as key enzymes. Both MDH1 and MDH2 expression levels in lung and spleen are low compared to liver. The lowest level of MDH1 expression was observed in human lung and bone marrow (Lo et al., 2005).

In conclusion, the differences in MDH activity between species are independent of the structure of the enzyme itself; however, these differences may depend on the regulation of expression, activity, or both. The distribution study showed that the level of MDH transcription correlates with the cellular energy requirement of the tissue. In addition, the enzyme activities are presumably regulated by lysine acetylation in the short term. Additional studies are required to clarify the mechanisms by which hormones and nutritional status regulate the activities, expression, and acetylation levels of MDHs in cats. 


\section{ACKNOWLEDGMENTS}

Research supported by the Nippon Veterinary and Life Science University. We thank Professor Toshiro Arai (Director of Laboratory of Veterinary Biochemistry, Nippon Veterinary and Life Science University) for providing laboratory space, equipment, and useful suggestions.

\section{REFERENCES}

Birktoft JJ, Fu Z, Carnahan GE, Rhodes G, et al. (1989). Comparison of the molecular structures of cytoplasmic and mitochondrial malate dehydrogenase. Biochem. Soc. Trans. 17: 301-304.

Bookout AL and Mangelsdorf DJ (2003). Quantitative real-time PCR protocol for analysis of nuclear receptor signaling pathways. Nucl. Recept. Signal. 1: e012.

Choudhary C, Kumar C, Gnad F, Nielsen ML, et al. (2009). Lysine acetylation targets protein complexes and co-regulates major cellular functions. Science 325: 834-840.

Claros MG and Vincens P (1996). Computational method to predict mitochondrially imported proteins and their targeting sequences. Eur. J. Biochem. 241: 779-786.

Falquet L, Pagni M, Bucher P, Hulo N, et al. (2002). The PROSITE database, its status in 2002. Nucleic Acids Res. 30: 235-238.

Gibson N and McAlister-Henn L (2003). Physical and genetic interactions of cytosolic malate dehydrogenase with other gluconeogenic enzymes. J. Biol. Chem. 278: 25628-25636.

Gietl C (1992). Partitioning of malate dehydrogenase isoenzymes into glyoxysomes, mitochondria, and chloroplasts. Plant Physiol. 100: 557-559.

Goward CR and Nicholls DJ (1994). Malate dehydrogenase: a model for structure, evolution, and catalysis. Protein Sci. 3: $1883-1888$.

Hall MD, Levitt DG and Banaszak LJ (1992). Crystal structure of Escherichia coli malate dehydrogenase. A complex of the apoenzyme and citrate at 1.87 A resolution. J. Mol. Biol. 226: 867-882.

Joh T, Takeshima H, Tsuzuki T, Setoyama C, et al. (1987). Cloning and sequence analysis of cDNAs encoding mammalian cytosolic malate dehydrogenase. Comparison of the amino acid sequences of mammalian and bacterial malate dehydrogenase. J. Biol. Chem. 262: 15127-15131.

Kelly CA, Nishiyama M, Ohnishi Y, Beppu T, et al. (1993). Determinants of protein thermostability observed in the 1.9-A crystal structure of malate dehydrogenase from the thermophilic bacterium Thermus flavus. Biochemistry 32: 3913-3922.

Kim EY, Kim WK, Kang HJ, Kim JH, et al. (2012). Acetylation of malate dehydrogenase 1 promotes adipogenic differentiation via activating its enzymatic activity. J. Lipid Res. 53: 1864-1876.

Kim SC, Sprung R, Chen Y, Xu Y, et al. (2006). Substrate and functional diversity of lysine acetylation revealed by a proteomics survey. Mol. Cell 23: 607-618.

Lo AS, Liew CT, Ngai SM, Tsui SK, et al. (2005). Developmental regulation and cellular distribution of human cytosolic malate dehydrogenase (MDH1). J. Cell. Biochem. 94: 763-773.

MacDonald ML, Rogers QR and Morris JG (1984). Nutrition of the domestic cat, a mammalian carnivore. Annu. Rev. Nutr. 4: 521-562.

Magori E, Nakamura M, Inoue A, Tanaka A, et al. (2005). Malate dehydrogenase activities are lower in some types of peripheral leucocytes of dogs and cats with type 1 diabetes mellitus. Res. Vet. Sci. 78: 39-44.

McAlister-Henn L (1988). Evolutionary relationships among the malate dehydrogenases. Trends Biochem. Sci. 13: 178181.

Minárik P, Tomásková N, Kollárová M and Antalik M (2002). Malate dehydrogenases - structure and function. Gen. Physiol. Biophys. 21: 257-265.

Mori A, Lee P, Takemitsu H, Sako T, et al. (2009). Comparison of insulin signaling gene expression in insulin sensitive tissues between cats and dogs. Vet. Res. Commun. 33: 211-226.

Musrati RA, Kollárová M, Mernik N and Mikulásová D (1998). Malate dehydrogenase: distribution, function and properties. Gen. Physiol. Biophys. 17: 193-210.

Scholz TD, Koppenhafer SL, Teneyck CJ and Schutte BC (1998). Ontogeny of malate-aspartate shuttle capacity and gene expression in cardiac mitochondria. Am. J. Physiol. 274: C780-C788.

Wang Q, Zhang Y, Yang C, Xiong H, et al. (2010). Acetylation of metabolic enzymes coordinates carbon source utilization and metabolic flux. Science 327: 1004-1007. 
Washizu T, Nakamura M, Izawa N, Suzuki E, et al. (2002). The activity ratio of the cytosolic MDH/LDH and the isoenzyme pattern of LDH in the peripheral leukocytes of dogs, cats and rabbits. Vet. Res. Commun. 26: 341-346.

Zhao S, Xu W, Jiang W, Yu W, et al. (2010). Regulation of cellular metabolism by protein lysine acetylation. Science 327: 1000-1004.

Zini E, Franchini M, Osto M, Vogtlin A, et al. (2010). Quantitative real-time PCR detection of insulin signalling-related genes in pancreatic islets isolated from healthy cats. Vet. J. 183: 287-293. 